\title{
Analysis of Perioperative Complications of Surgery for Proximal Femur Fracture in Elderly Patients Older than 75 Years
}

\author{
Tsuyoshi Nakai*, Kunihiko Hashimoto, Atsunori Onishi, Toshiyuki Nakamura, Akira Miyama, \\ Naohiro Yasuda
}

Department of Orthopaedic Surgery, Itami City Hospital, Itami, Japan.

Email: "tsuyoshi223@gmail.com

Received March 28 ${ }^{\text {th }}, 2013$; revised May $3^{\text {rd }}, 2013$; accepted May $20^{\text {th }}, 2013$

Copyright (c) 2013 Tsuyoshi Nakai et al. This is an open access article distributed under the Creative Commons Attribution License, which permits unrestricted use, distribution, and reproduction in any medium, provided the original work is properly cited.

\begin{abstract}
Background: In elderly patients with femoral neck fracture, functional recovery is affected largely by preoperative underlying diseases and postoperative complications. The present study was designed to analyze the relationship between preoperative underlying diseases and perioperative complications of surgery for proximal femur fracture. Methods: The study involved 86 patients aged 75 years or older, who underwent surgery for proximal femur fracture. Each patient was examined for the presence of preoperative underlying diseases and preoperative biochemical test data. Moderate and severe complications, which were life-threatening and necessitated treated, were investigated during the perioperative period. A retrospective analysis was conducted on the relationship of preoperative underlying diseases and preoperative biochemical data with the moderate or severe complications that developed during the perioperative period. Results: Eighty-one patients developed a preoperative underlying disease, with hypertension being most frequent (42 cases). Complications during the perioperative period were observed in 58 patients, with anemia develops most frequently (23 cases). Moderate or severe complications during the perioperative period were observed in 16 patients, and infection was the most frequent complication (8 cases). In the statistical analysis, a significant difference in the incidence of moderate or severe perioperative complications was observed only in the users of anticoagulants/antiplatelet drugs. Discussion: Patients of preoperative use of anticoagulants/antiplatelet drugs should be closely managed medically so that perioperative medical complications can be managed.
\end{abstract}

Keywords: Perioperative Complications; Proximal Femur Fracture; Elderly Patients

\section{Introduction}

Following the recent trend of aging of the population in Japan, the annual incidence rate of proximal femur fracture has been increasing every year, recording 148,100 cases in 2007 [1]. In many cases, hip fractures are a sentinel event signaling a systemic decline in the patient's health. Elderly people tend to have compromised function and low reserve capabilities of organs and are therefore likely to develop various complications during the perioperative period. Among others, the risk of death from perioperative complications has been reported in elderly patients with femoral neck fracture. The present study was undertaken to analyze the association between preoperative underlying diseases and perioperative complications of surgery for proximal femur fracture in eld-

*Corresponding author. erly patients older than 75 years.

\section{Materials and Methods}

The study involved 86 patients aged 75 years or older, who underwent surgery for proximal femur fracture at our hospital between January 2010 and June 2011. Inclusion criteria of this study were 1) hip fracture patients operated in our hospital; 2) age older than 75 years; Japanese patients; and 3) at least 12 months of follow-up, except for patients with lethal events. Exclusion criteria were cases of pathological fracture. There were 18 men and 68 women, with ages ranging from 75 to 98 years (mean, 85.8 years). The femur fracture was a medial neck fracture in 48 cases and a trochanteric fracture in 38 cases. The operative procedure applied was invasive reduction and fixation in 53 cases, prosthetic femoral neck 
Table 1. Demographic data.

\begin{tabular}{lc}
\hline Characteristic & \\
No. of patients & 86 \\
Age (years) & 85.8 \\
Sex & \\
Male & 18 \\
Female & 68 \\
Fracture type & \\
Neck fracture & 48 \\
Trochanteric fracture & 38 \\
Operative procedures & \\
Reduction and fixation & \\
Prosthetic femoral neck replacement & 53 \\
Total hip replacement & 31 \\
& 2 \\
\hline
\end{tabular}

replacement in 31 cases, and total hip replacement in 2 cases (Table 1). Each patient was examined for the presence of preoperative underlying diseases, including hypertension, cardiovascular disease, respiratory disease, gastrointestinal disease, diabetes mellitus, cerebrovascular disease, malignant disease, and dementia, and the use of anticoagulants/antiplatelet drugs. In addition, the patients' medical records were reviewed for any history of orthopedic diseases (proximal femur fracture and compression fracture of the spine). Preoperative biochemical test data (hemoglobin, albumin, blood urea nitrogen [BUN], creatine [Cre], aspartate aminotransferase [AST], alanine aminotransferase [ALT], $\mathrm{Na}, \mathrm{K}$, and Ca levels) were also obtained. Moderate and severe complications, which were life-threatening and necessitated treatment, were investigated during the perioperative period. A retrospective analysis was conducted on the relationship of preoperative underlying diseases and preoperative biochemical data with the moderate or severe complications that developed during the perioperative period (from admission to the third postoperative week). The chi-square test was used for statistical analysis.

\section{Results}

Eighty-one patients developed a preoperative underlying disease, with hypertension being most frequent (42 cases), followed by cerebrovascular disease (27 cases) and anemia (27 cases), as shown in Table 2. Complications during the perioperative period were observed in 58 patients, with anemia developing most frequently (23 cases), followed by psychiatric disorders (17 cases) and infection (17 cases). Moderate or severe complications during the perioperative period were observed in 16 patients, and infection was the most frequent complication (8 cases), followed by respiratory and cardiovascular diseases. The disease that most frequently occurred as a complication was aspiration pneumonia (4 cases). One patient died during the perioperative period (Table 3). In the analysis of the relationship between preoperative underlying diseases and moderate or severe perioperative complications, the incidence rate of moderate or severe complications was highest in the patients with malnutrition as the preoperative underlying disease $(42.9 \%)$ and second highest in the patients with nephropathy (41.7\%). The number of cases of moderate or severe perioperative complications was largest among users of anticoagulants/antiplatelet drugs (9 cases; Table 4). In the statistical analysis, a significant difference in the incidence of moderate or severe perioperative complications was observed only in the users of anticoagulants/antiplatelet drugs. The presence of underlying nephropathy also showed a similar

Table 2. Preoperative underlying diseases.

\begin{tabular}{lc}
\hline \multicolumn{1}{c}{ Underlying disease } & No. of Cases \\
\hline Hypertension (under treatment) & 42 \\
Previous cerebrovascular disease & 27 \\
Anemia $(\mathrm{Hb}<10.0 \mathrm{~g} / \mathrm{dL})$ & 27 \\
Orthopedic disease & 26 \\
Use of anticoagulants/antiplatelet drugs & 26 \\
Previous cardiovascular disease & 25 \\
Gastrointestinal disease (treated surgically) & 23 \\
Previous respiratory disease & 20 \\
Diabetes mellitus (under treatment) & 20 \\
Dementia (DSM-IV diagnostic criteria) & 19 \\
Previous malignant disease & 13 \\
Nephropathy (BUN $>40$ mg/dL, Cre $>1.5$ mg/dL) & 12 \\
Malnutrition (Alb < 2.5 g/dL) & 7 \\
Hepatopathy (GOT $>50$, GPT $>50)$ & 6 \\
Electrolyte abnormality (under treatment) & 2 \\
\hline
\end{tabular}

Table 3. Perioperative complications.

\begin{tabular}{lc}
\hline \multicolumn{1}{c}{ Complication } & No. of cases \\
\hline Blood transfusion & $23(2)$ \\
Psychiatric disorders (delirium onset/aggravation) & 17 \\
Infection & $17(8)$ \\
Respiratory disease & $8(7)$ \\
Cardiovascular disease & $8(3)$ \\
Hepatopathy & 8 \\
Gastrointestinal disease & $6(1)$ \\
Nephropathy & 5 \\
Cerebrovascular disease & $2(2)$ \\
Electrolyte abnormality & 1 \\
Death & 1 \\
\hline
\end{tabular}

The values in the parentheses indicate the number of moderate or severe cases of complication. 
Table 4. Association between preoperative underlying disease and moderate or severe complications.

\begin{tabular}{lc}
\hline \multicolumn{1}{c}{ Preoperative underlying disease } & $\begin{array}{c}\text { Moderate/severe } \\
\text { complication }\end{array}$ \\
\hline Malnutrition (Alb $<2.5 \mathrm{~g} / \mathrm{dL})$ & $\begin{array}{c}\text { incidence rate } \\
\text { (no. of cases) }\end{array}$ \\
Nephropathy (BUN $>40 \mathrm{mg} / \mathrm{dL}$, & $42.9 \%(3)$ \\
Cre > 1.5 mg/dL) & $41.7 \%(5)$ \\
Use of anticoagulants/antiplatelet drugs & $34.6 \%(9)$ \\
Hepatopathy (GOT > 50, GPT > 50) & $33.3 \%(2)$ \\
Gastrointestinal disease (treated surgically) & $30.4 \%(7)$ \\
Diabetes mellitus (under treatment) & $30 \%(6)$ \\
Previous cardiovascular disease & $28 \%(7)$ \\
Hypertension (under treatment) & $26.2 \%(11)$ \\
Previous respiratory disease & $25 \%(5)$ \\
Previous malignant disease & $23.1 \%(3)$ \\
Anemia (Hb $<10.0$ g/dL) & $22.2 \%(6)$ \\
Dementia (DSM-IV diagnostic criteria) & $21 \%(4)$ \\
Orthopedic disease & $19.2 \%(5)$ \\
Cerebrovascular disease (history) & $14.8 \%(4)$ \\
Electrolyte abnormality (under treatment) & $0 \%(0)$ \\
\hline
\end{tabular}

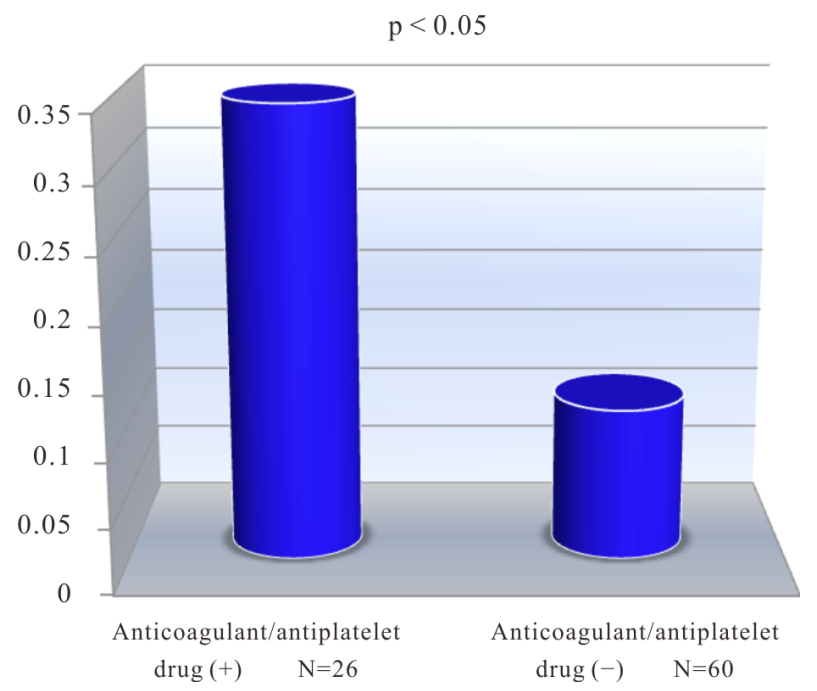

Figure 1. Incidence of moderate or severe complications.

tendency regarding the influence on the incidence of moderate or severe complications, although this tendency was not statistically significant $(\mathrm{P}=0.07)$. The other preoperative underlying diseases did not significantly affect the incidence of complications (Figure 1).

\section{Discussion}

It was previously reported that elderly patients with this type of fracture often had preoperative underlying dis- eases [2-4]. Among the patients managed at our facility, $94 \%$ had underlying diseases before surgery. Complications known to develop frequently after surgical treatment of proximal femur fracture include psychiatric disorders, pneumonia, and cardiac disease [2,4,5]. In our cases, anemia requiring blood transfusion was the most frequent complication, followed by psychiatric disorders and infection. One possible reason why many cases in the present study required perioperative blood transfusion is that there were no evident criteria for administering blood perfusion and the decision was at the discretion of each physician. Reports are available regarding the association between preoperative underlying diseases, such as cardiac disease, respiratory disease, psychiatric disorders, malnutrition, renal function, and previous multiple diseases, and moderate or severe perioperative complications [6,7]. In our cases, a significant difference in the incidence of moderate or severe perioperative complications was observed only in users of anticoagulants/antiplatelet drugs. This finding may be possibly explained by our results indicating that of all anticoagulant/antiplatelet drug users, 92.3\% (24/26 cases) had multiple preoperative underlying diseases and 65.3\% (17/26 cases) had a history cardiovascular or respiratory disease.

\section{Conclusion}

Among the 86 patients with proximal femur facture, the relationship between preoperative underlying diseases and perioperative complications was analyzed. Preoperative underlying diseases and perioperative complications were observed in 81 and 58 patients, respectively. Moderate or severe perioperative complications were observed in 16 patients. Preoperative use of anticoagulants/ antiplatelet drugs correlated significantly with perioperative development of moderate or severe complications. When elderly patients, aged 75 years or older, have a proximal femur fracture and are preoperatively believed to have a high risk of perioperative complications, the patients themselves and their family members should be informed well about the preventive measures that should be undertaken to avoid perioperative complications.

\section{REFERENCES}

[1] National Livelihood Survey, "The Ministry of Health, Labour and Welfare,” Japan, 2007.

[2] A. Kudo, A. Masuoka and A. Matsuki, "Analysis of PeRioperative Complications in Patients with Femoral Neck Fracture Aged over 80," Orthopedic Surgery, Vol. 44, 1993, pp. 1858-1861.

[3] K. Sakamoto, T. Nakamura, H. Hagino, N. Endo, S. Mori, Y. Muto, A. Harada, S. Yamamoto, K. Kushida, M. Yo- 
shimura and H. Yamamoto, "Report on the Japanese Orthopaedic Association's 3-Year Project Observing Hip Fracture at Fixed-Point Hospitals,” Journal of Orthopaedic Science, Vol. 11, No. 2, 2006, pp. 127-134. doi:10.1007/s00776-005-0998-1

[4] Y. Asao, T. Higuchi and Y. Shimoda, "Analysis of PeRioperative Complications among 449 Elderly Patients with Femoral Neck Facture Treated Surgically,” Journal of Clinical Anestheology, Vol. 25, 2005, p. 323.

[5] S. Kawahara, K. Sato, T. Ando, S. Kitamura and K. Miyasaka, "Perioperative Complications and Their Management in Patients with Femoral Neck Fracture in Late
Senility,” Central Japan Journal of Orthopaedic Traumtology, Vol. 45, 2002, pp. 871-872.

[6] K. Ichimura and T. Ishii, "Prognosis of Elderly Patients with Proximal Femur Fracture Accompanied by Medical Disease," Central Japan Journal of Orthopaedic Traumatology, Vol. 45, 2002, pp. 861-862.

[7] K. Otani and Y. Inomata, "Perioperative Complications and Prognosis as to Survival in Elderly Patients with Femoral Neck Facture,” Journal of the Eastern Japan Association of Orthopaedics and Traumatology, Vol. 15, 2003, pp. 569-574. 